Publicación semestral. ISSN 2215-4906

Volumen 81 - Número 1

Revista de las artes Julio - Diciembre 2021

\title{
Pensar a Simón Rodríguez desde las prácticas artísticas: La indisciplina como una forma de resistencia afectiva
}

Thinking Simón Rodríguez from artistic practices:

Indiscipline as an affective resistance

\author{
Rubén Cerrillo
}

DOI 10.15517/es.v81i1.47647

(c) (i) (9) Esta obra está bajo una licencia Creative Commons
BY 


\title{
Pensar a Simón Rodríguez desde las prácticas artísticas: La indisciplina como una forma de resistencia afectiva
}

Thinking Simón Rodríguez from artistic practices: Indiscipline as an affective resistance

\author{
Rubén Cerrillo ${ }^{1}$ \\ Universidad Nacional Autónoma de México, \\ Ciudad de México, México
}

Recibido: 12 de febrero de 2021 Aprobado: 17 de marzo de 2021

\section{Resumen:}

Este artículo busca desarrollar una relatoría sentipensante de lo que ha implicado para el Mitote ICDAC revisar el pensamiento de Simón Rodríguez desde dos aristas fundamentales: En primer lugar, desde sus postulados de Educación Popular, los cuales eran abiertamente decoloniales en el momento de la Colonia y muchísimo antes de que se acuñara el término como matriz filosófica, el cual nos sirve como raíz y tierra fértil para re-pensar las dimensiones de lo que implica actuar desde la Universidad Pública, y permite la articulación espacio posible para trabajar desde un paradigma indisciplinado ante la hegemonía del conocimiento jerarquizado. En segundo lugar, se propone pensarlo desde las prácticas artísticas como una forma de ejercer la autonarración situada, como un medio que nos faculta para hacer/ pensar las preguntas que nos atraviesan como forma de estar en el mundo y con ello poder pensarlo en nuestros propios términos, ya que es una de las invitaciones fundamentales en la obra de Rodríguez.

Palabras clave: arte, comunidad, educación, América Latina, colonialismo, investigación

1 Profesor asociado en la Facultad de Artes y Diseño en la Universidad Autónoma de México. Maestro en Artes Visuales, Universidad nacional Autónoma de México. ORCID: 0000-0003-2848-9074. Correo electrónico: rcerrillo@fad.unam.mx 
Pensar a Simón Rodríguez desde las prácticas artísticas:

\begin{abstract}
:
This paper seeks to develop a sentimental account of what it has implied for the Mitote ICDAC to review the thought of Simón Rodríguez from two fundamental points. First from his postulates about Popular Education, which was openly decolonial at the time of the Colony and much earlier that the term was coined as a philosophical matrix, which serves as a root and fertile ground to re-think the dimensions of what it means to act from the Public University, and articulate it as a possible space to act from an undisciplined paradigm in the face of the hegemony of knowledge. Second, think about it from artistic practices as a way of exercising situated self-narration, as a means that empowers us to ask/think the questions that cross us as a way of being in the world and with it to be able to think about it in our own terms, what it is one of the fundamental invitations in Rodríguez's work.
\end{abstract}

Keywords: art, community, education, Latin America, colonies, research 


\section{Llamado al mitote Rodriguista}

Nuestras primeras palabras para este escrito² están tejidas desde los imaginarios de tono gustoso que los textos y las charlas en torno a la figura de Simón Rodríguez han detonado en el Mitote ICDAC. Decimos tono gustoso derivado de dos términos, el primero, es el de Xavier Zubiri:

Un sentimiento no es, como vulgarmente se piensa, un afecto o una afección (aunque esto tiene su justificación, pero estrictamente no es eso). Afección es ... una cosa que uno sufre, que uno padece, no en el sentido de dolor, pero sí que uno recibe. Esto no es forzosamente un sentimiento. Esto, sin más es una modificación del tono vital, que el hombre como todo animal puede tener, y que en tanto que animal tiene. El momento específico y propio del sentimiento consiste en que aquello que modifica nuestro tono vital no sea estímulo sino una realidad. (Zubiri, 1993, p. 353)

El segundo, es en el sentido de la lectura que Enrique Dussel refiere en su análisis sobre el tono de Zubiri, llevado a temas del gusto, donde aclara que

lo que me gusta es porque tiene la capacidad, en último término de reproducir mi vida, pero no como conocimiento, no como decidir hacer eso para lograr algo, que es la voluntad, sino el tocar la cosa misma y descubrir en su sustancialidad que es una mediación para la afirmación de la vida del sujeto... lo que gusto no es solo una mediación, es la realidad misma de la cosa (Dussel, 2020, 1h 03m 22s).

Este tono gustoso nos ha permitido que en, desde y a partir del grupo de investigación se construyan reflexiones, diálogos, consensos y disensos respecto de las rimas conceptuales de las prácticas emancipadoras y subversivas que Rodríguez articulaba, desde sus proposiciones filosóficas en el siglo XIX. Estas pueden reflejarse en las prácticas artísticas que nosotrxs ${ }^{3}$ habitamos como forma de resistencia en nuestras aulas y espacios académicos, con una vigencia, por un lado, abrumadora y, por el otro, reconciliante, pues nos permite tejerlas con todas esas voces amigas que nos acuerpan en nuestras rebeldías epistémicas.

2 Este es un trabajo realizado con el apoyo del Programa UNAM-DGAPA-PAPIME PE404520.

${ }^{3}$ Nota editorial: en este artículo se respeta la posición del autor con respecto al uso de la ' $x$ ' como marcador inclusivo, a pesar de que esta forma no se encuentra dentro del uso normativo del español ni se apega a las políticas de lenguaje inclusivo de la institución editora. 
Pensar a Simón Rodríguez desde las prácticas artísticas:

Dossier

La indisciplina como una forma de resistencia afectiva

Imagen 1. Voces amigas tejidas/ $11^{\circ}$ Jornada Rodriguista

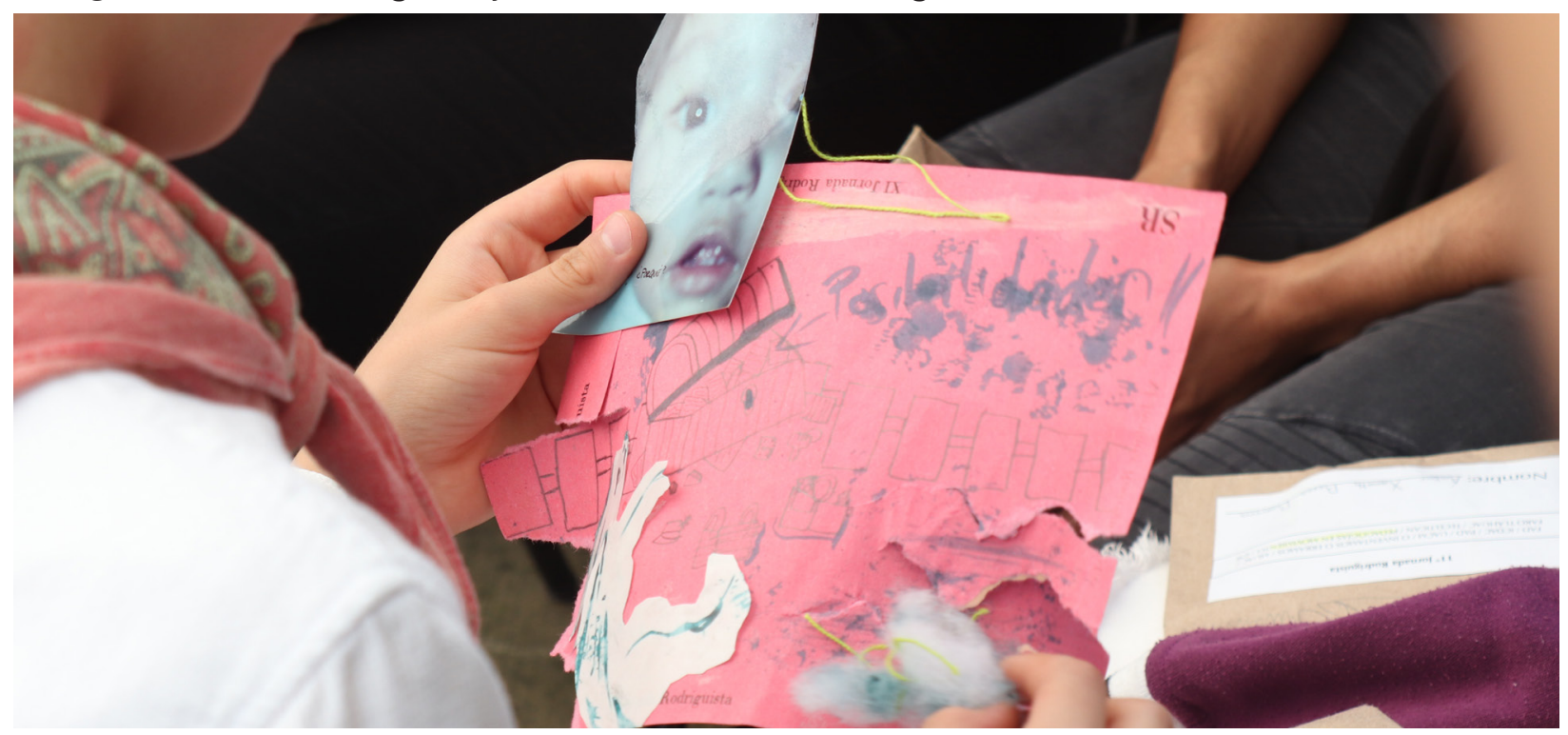

Fuente: Mitote ICDAC

Tomemos, por ejemplo, uno de los nodos imaginarios que hemos definido para sentarnos a dialogar con y desde Rodríguez. Este nodo está articulado desde la idea establecida en su PRODROMO sobre la necesidad de "pintar los pensamientos bajo la forma en que se conciben... (en la estructura de estas pájinas se ve el ejemplo) en el modo de pintar consiste la expresión, y por la expresión se distinguen los estilos" (Rodríguez, 1828/2018, p. 25). Es desde esa necesidad por pintar los pensamientos, en la forma en la que los concebimos, que nos incluimos e incluimos a todxs Ixs interesadxs en vernos cercanxs a estas ideas. Lo planteamos con esa " $x$ " que expresa nuestra intención de acuerpar las luchas y las justicias epistémicas ligadas a las exigencias por la autonomía auto-representacional de todxs aquellxs quienes han sido negadxs por las violencias de género encarnadas en las prácticas discursivas, las cuales sostienen la discriminación sistémica hacia quienes no entramos y no queremos entrar en los lugares asignados por el discurso heteropatriarcal.

Por esta misma razón es que hablamos en plural. Creemos en la posibilidad electiva de suscribirnos a las acciones conceptuales, expresivas y declarativas de aquellos que volvemos nuestrxs al convertirnos en ese nosotrxs. A partir de este nos podemos enunciar con fuerza, como afectos a los que nos vinculamos, y no como cosas ligadas a la propiedad privada, desde la ternura radical, a la manera de lo enunciado por Dani d'Emilia y Daniel B. Chávez en su Manifiesto vivo: 
ternura radical es aceptar lo ambiguo

es no pensar dándole vueltas a tu ombligo

es romper con patrones afectivos, sin expectativas claras

ternura radical es compartir sueños, locura

sintonizar, no solo empatizar

es encontrar una galaxia en los ojos de otrx y no dejar de mirar

es leer el cuerpo del otrx como un palimpsesto

ternura radical es canalizar energías irresistibles

y convertirlas en encarnaciones indominables (D’Emilia \& Chávez, 2015, versos 37-45)

Imagen 2. Herramientas para todxs/ El gran Mitote con la Novia Sirena

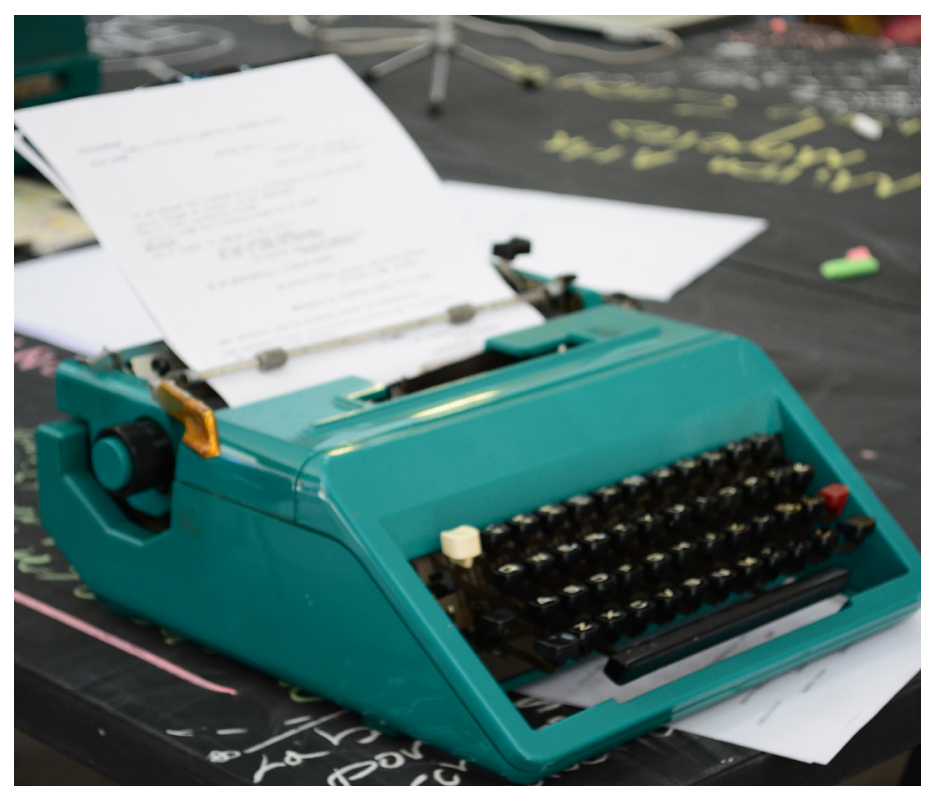

Fuente: Mitote ICDAC
Creemos que todxs los que vivimos en este cotidiano, somos Ixs que sembramos la discusión que puede germinar los frutos que nos interesan y significan en este recorrido vivo. Coincidimos totalmente con las posturas de Iván Illich quien, como lo refieren Jean Robert y Valentina Borremans en el prefacio a sus Obras Reunidas I "quería alentar una investigación liberada del monopolio radical de los expertos, una investigación hecha por la gente" (Illich, 2006, p. 21). Por ello, en estas líneas hay una enunciación clara de la multiplicidad y colaboración entre voces, formas de pensamiento y las posibilidades de hacer/tejer juntxs.

Para conversar es necesario situarnos y disponernos con verdadero ánimo de abrirnos a la experiencia situada de Ixs otrxs, con ganas de conocer y significar los saberes 
Pensar a Simón Rodríguez desde las prácticas artísticas:

Dossier

La indisciplina como una forma de resistencia afectiva

que provienen de otras vidas, las cuales son posibles y valiosas por el hecho mismo de que fueron vividas por personas con sueños, luchas y resistencias. Aquí, lo hacemos desde las voces, en su tejido colectivo, las cuales nos disponen a configurar una mirada que nos permita invocar a Simón Rodríguez para dialogar sobre sus ideas y sus condiciones desde las claves permitidas por las prácticas artísticas, como una herramienta conceptual para desorganizarnos en el mundo, para desmarcarnos de los lugares asignados que la historia y la disciplina nos han impuesto. Por eso, des-obedecemos la idea de muro epistémico, como cosa que limita, separa y divide bajo sus supuestos de pertinencia disciplinar. Abrazados a la metáfora del rincón que Lia García nos regala desde su pensamiento tejido en la ternura radical, hablaremos con Simón desde el pequeño rincón sentipensante que articula este texto, pues como dice Lia "[q]ué sería de la posibilidad del rincón, de arrinconarnos, sin saber que un rincón es el punto, la esquina, el recoveco donde dos muros... se están besando" (Afrontera, 2020, 7 min $26 \mathrm{seg}$ ).

Desde aquí, proponemos que leer a Rodríguez desde las prácticas artísticas implica, de entrada, tener claro que, si bien el área disciplinar a la que se le ha adscrito a él y a sus ideas es la filosofía, en su "ADVERTENCIA" encontramos pistas para ligarlo a una reflexión bajo criterios que hoy denominaríamos transversales, nos dice que

[e]l estado actual de la América pide serias reflexiones... aprovechen los Americanos la Libertad de Imprenta que se han dado, para consultarse sobre el importante negocio de su libertad... El autor de esta obra ha procurado reunir pensamientos en favor de la causa social. (Rodríguez, 1828/2018, s/p)

Su reflexión apela a prácticas que provienen de experiencias y miradas múltiples, que invitan a la acción crítica y, sobre todo, rebelde, con la certeza de que en su contexto hay estrategias argumentativas que enmascaran y desvían la acción reflexiva y dialéctica. Tal como lo como argumenta en el mismo texto «iCuántos hombres, tal vez menos recomendables que el autor, no se ponen detrás de las cosas sagradas para valer algo!» (Rodríguez, 1828/2018, s/p)

Al decir lo anterior queremos mostrar que en Rodríguez hay una sapiencia que no se delimita, bajo ninguna circunstancia, a un campo especializado de estudio, a una disciplina, sino más bien a una experiencia de vida. Esta busca ser pensada y enunciada de manera

${ }^{4}$ Las mayúsculas de los títulos de los textos, así como de las citas textuales de Rodríguez se respetan del original por decisión del autor.

ESCENA. Revista de las artes, 2021, Vol. 81, Núm. 1 (julio-diciembre), pp. 282-303 
profunda desde ahí, desde el acontecimiento y no desde las prefiguraciones conceptuales, con todas las dudas y las indefiniciones que esto detone, cuyo resultado va construyendo propuestas epistémicas de rebeldía radical, como se advierte en aquella cita que es célebre entre aquellxs que nos encontramos en sus palabras, dónde se pregunta: "Dónde iremos a buscar modelos?...La América Española es orijinal = ORIJINALES han de ser sus Instituciones i su Gobierno= y ORIJINALES los medios de fundar uno i otro." O inventamos o Erramos (Rodríguez, 2018, p. 47).

Imagen 3. Conectando voces/ Mitote Simon Rodríguez

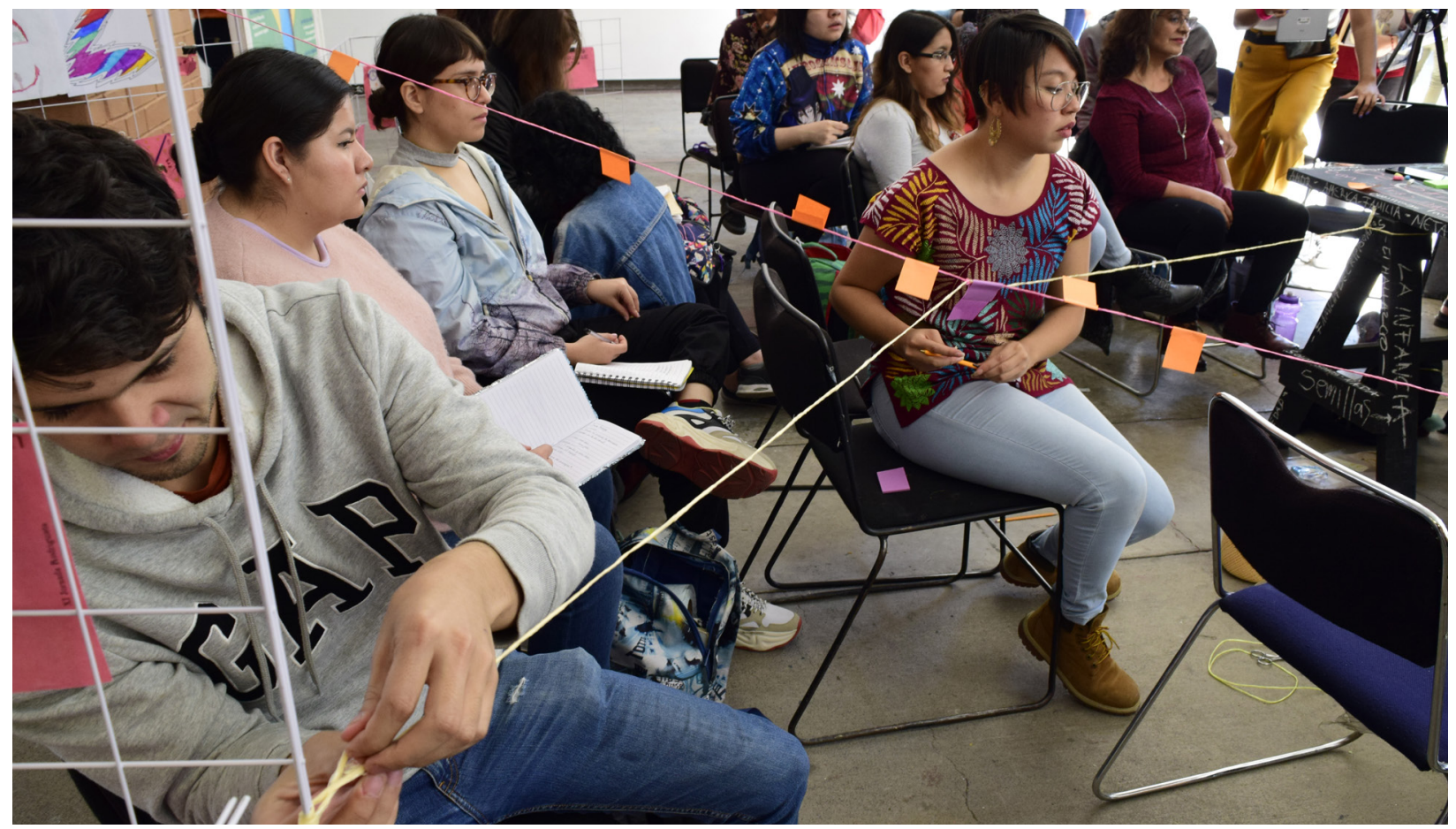

Fuente: Mitote ICDAC

Encontramos en su pensamiento des-obediente, ligado a su práctica de impresor, educadory hacedor develas, una experienciasituada. Esto nos permite tejerlo con la esencia de las prácticasartísticas, ya queestasactivan, como partedesu proceso creativo/reflexivo, formas metodológicas propias, al cuestionar, demaneracríticay específica, la pertinenciaysignificación que las técnicas, soportes, ideas, declaraciones, validadores y condicionantes tienen sobre las acciones que nos acompañan en los procesos vivos ligados a crear desde el arte. Entendemos el arte como una práctica que, alejada de la idea de consumo y productividad, es una acción 
Pensar a Simón Rodríguez desde las prácticas artísticas:

Dossier

La indisciplina como una forma de resistencia afectiva

que, desde nuestras posturas declaradas en el hacer, nos invita a sentirnos menos solxs en un sistema que ha trabajado con ahínco en separarnos del cosmos, entendido este último como "la totalidad de la realidad antes de la existencia del ser humano" como lo explica Dussel en su curso de Estética de la liberación. (Dussel, 2020, 3m 50s)

Imagen 4. Palabras vela/ $11^{\text {a }}$ Jornada Rodriguista

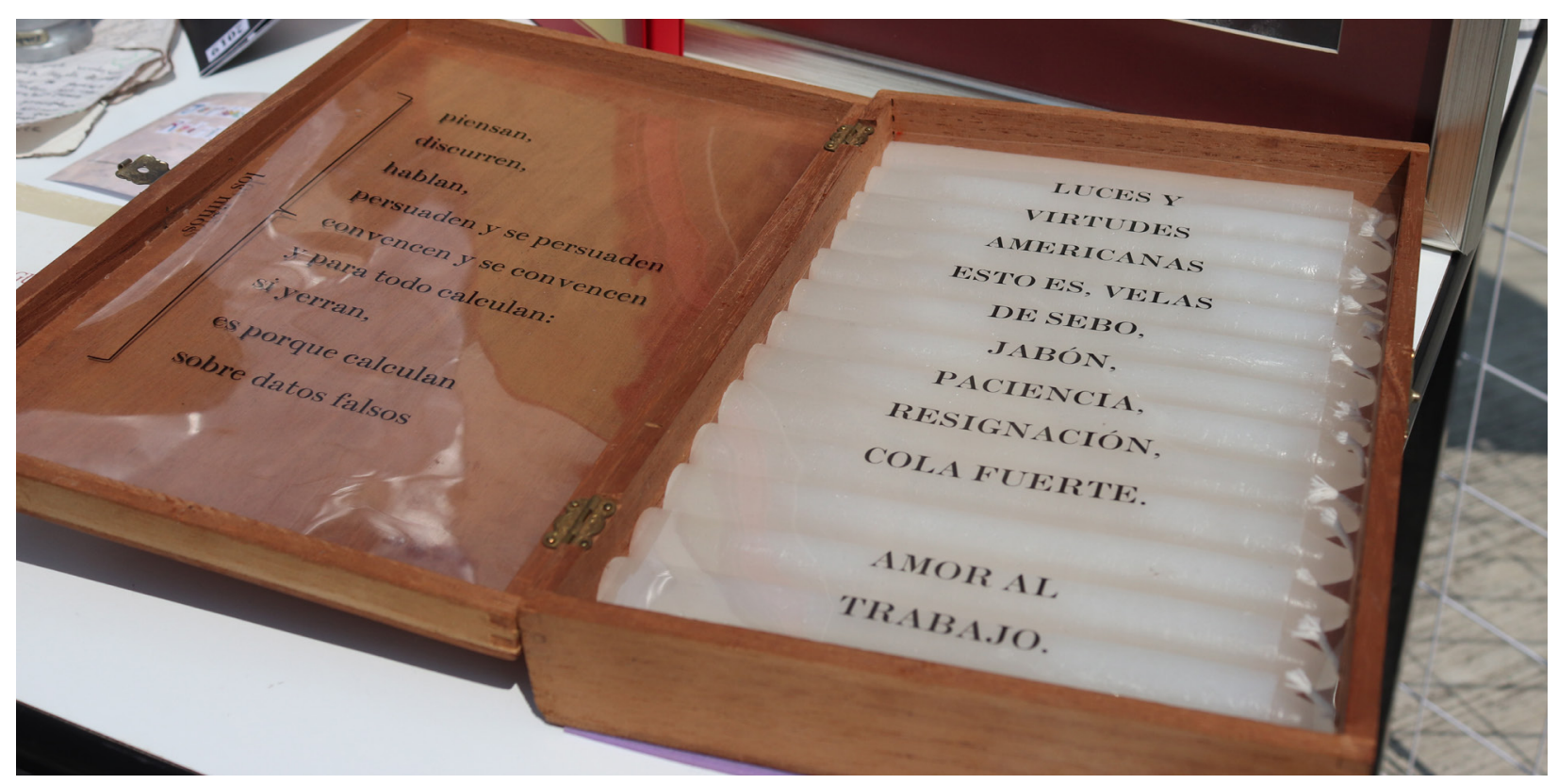

Fuente: Mitote ICDAC

En las prácticas artísticas que ejecutamos se apela a la mediación con Ixs otrxs través de dispositivos que intentan detonar un vínculo corporal, como aquello que despierta sensaciones afectivas en los cuerpos que sienten y experimentan, sin explicarse necesariamente desde la razón. Una de las búsquedas es construir reflexiones que despierten una tensión entre empatía y sintonía respecto a los pensamientos complejos, enunciados desde narrativas que no partan de lógicas heteronormativas. Desde ahí, cuando las prácticas artísticas alcanzan su cometido es que logran que nos sintamos menos solxs, con suerte, hasta mucho más reflexivxs. Experiencias vivas, como la de Simón Rodríguez, nos han permitido integrar herramientas conceptuales normalmente descalificadas por el discurso hegemónico. Nociones como el error, la indeterminación, las violencias epistémicas o la gestión política de la subjetividad no solo son reflexiones legítimas, sino también una cuestión sustancial e integrada, lo que el Mitote ICDAC sostiene como una disidencia. 
Imagen 5. A la pizarra con los cuates/ Mitote Juan Acha

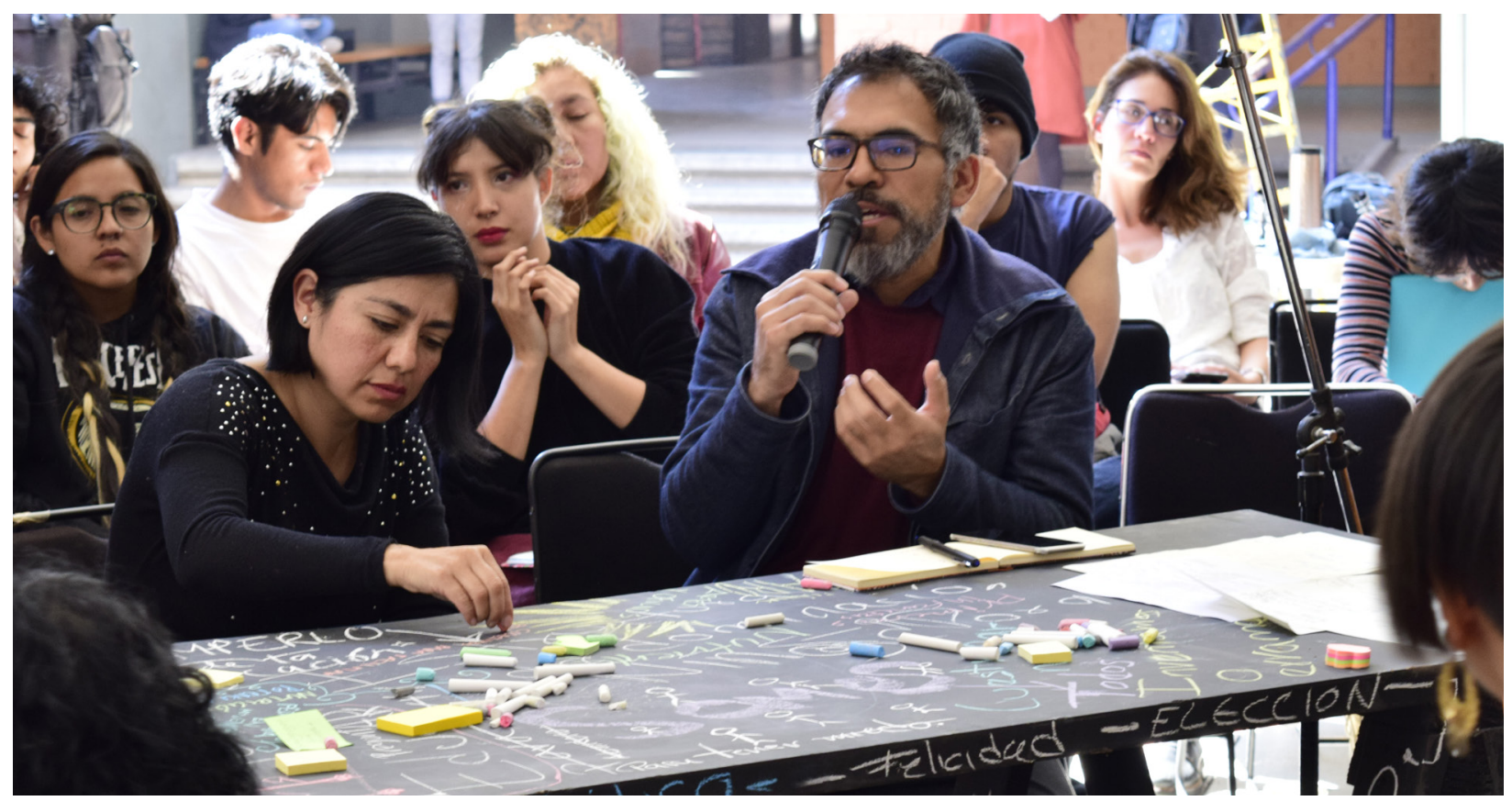

Fuente: Mitote ICDAC

La Estética de la liberación es entonces una práctica subversiva, entendido lo estético como "el lugar del enunciado consciente... de mi pensar" (Dussel, 2020, 2m 11s). En palabras de Dussel, en el desarrollo y complejización de nuestras búsquedas estéticas desde la práctica artística, y en realidad desde cualquier ejercicio de los saberes situados, se pueden construir herramientas enunciativas ligadas al conjunto de nuestros saberes, propios y comunitarios. Aunque esto pareciera menor, no lo es, pues la tecnificación, la parcelación disciplinar y obsesión con la hiper-especialización nos han llevado a replicar la educación heteronormativa y binaria.

Para entender a profundidad sus textos se requiere una ardua faena de lectura e interpretación, en la que es esencial considerar los estratos socio-económicos y políticos de la América del siglo XIX. Estos impactaban directamente en las propuestas educativas de Rodríguez y en cómo plasmaba en sus impresos discursos que buscaban no llegar a estratos privilegiados, sino a poblaciones que se identificaban con las situaciones enunciadas y puestas sobre el papel para conocimiento público. Es crucial mencionar que Rodríguez tenía una mirada hacia la educación popular, la cual veía desde su cotidiano. Nosotrxs hacemos lo mismo desde nuestro cotidiano, con otras formas de ver y vivir la educación. 
Pensar a Simón Rodríguez desde las prácticas artísticas:

Dossier

La indisciplina como una forma de resistencia afectiva

A partir de lo anterior, lo hacemos un compañero de trincheras y de andanzas al obtener del cotidiano herramientas para el aprendizaje. Nuestra forma de abordarlo es traerlo a nuestro contexto desde las múltiples metáforas de su hacer, para así, acercarnos a él como educador/filósofo/impresor/caminante.

Así lo hemos hecho durante tres años con el seguimiento y participación en las Jornadas Rodriguistas. Hemos jugado con la idea de pensar a Simón Rodríguez como un compañero que nos invita a ser rebeldes e indisciplinados ante la imposición hegemónica del pensamiento, en compañía de nuestras red de colaboración: el grupo de investigación O inventamos o erramos de la Universidad Autónoma de la Ciudad de México (UACM) Campus Del Valle; la Facultad de Artes y Diseño de la UNAM (FAD); el Movimiento Mexicano para la Educación Moderna (MMEM); la Fábrica de Artes y Oficios (F.A.R.O.) Tláhuac, y el grupo de investigación pedagógica Pedagogía en movimiento (Adriana López y Johnatan Rojas). A través de Rodríguez, nos vemos resistentes y afectivos ante un mundo que nos señala con urgencia que el porvenir o será comunitario o no será.

Vamos a empezar a partir de nuestra experiencia en la $11 .^{a}$ Jornada Rodriguista. Es indispensable aquí enunciar que en esta buscábamos resaltar la posibilidad de mirar la educación como una forma de emancipación de las estructuras hegemónicas de control sobre los sujetos. Así, se pretendía articular una pieza de arte procesual que permitiera realizar un trabajo significativo y colaborativo entre estudiantes de primaria, licenciatura y posgrado, tanto de sistemas de educación formal, no formal y activa. Este proyecto colaborativo entre la red anteriormente mencionada tenía mucho en juego. Primero, ser conscientes de la educación que estamos dando como docentes en cada una de nuestras aulas, cualquiera que sea el formato de ellas y suscritas a las jerarquías académicas de sus respectivas instituciones públicas. Segundo, construir juntxs un eje articulador sobre el cual practicar metodologías Rodriguistas. Por ello, retomamos las narraciones donde se cuenta que Simón Rodríguez solía trabajar en la misma aula con estudiantes de todas las edades y condiciones, sin distinción alguna, salvo tal vez la que su propia contextualidad demandaba y que, por tanto, era su materia de trabajo. Esto le servía, sobre todo, para, por primera vez en la vida de muchos, tener el permiso para preguntar, situación que nos parecía una oportunidad imponente y desbordada que un grupo de docentes podría proponer como acto de resistencia educativa frente a un sistema burocratizado, cuyo único objetivo es cuantificar los conocimientos en función de la producción en masa de obreros privatizados.

ESCENA. Revista de las artes, 2021, Vol. 81, Núm. 1 (julio-diciembre), pp. 282-303 
Imagen 6. Enseñad a los niños a ser preguntones/ $11^{\text {a }}$ Jornada Rodriguista

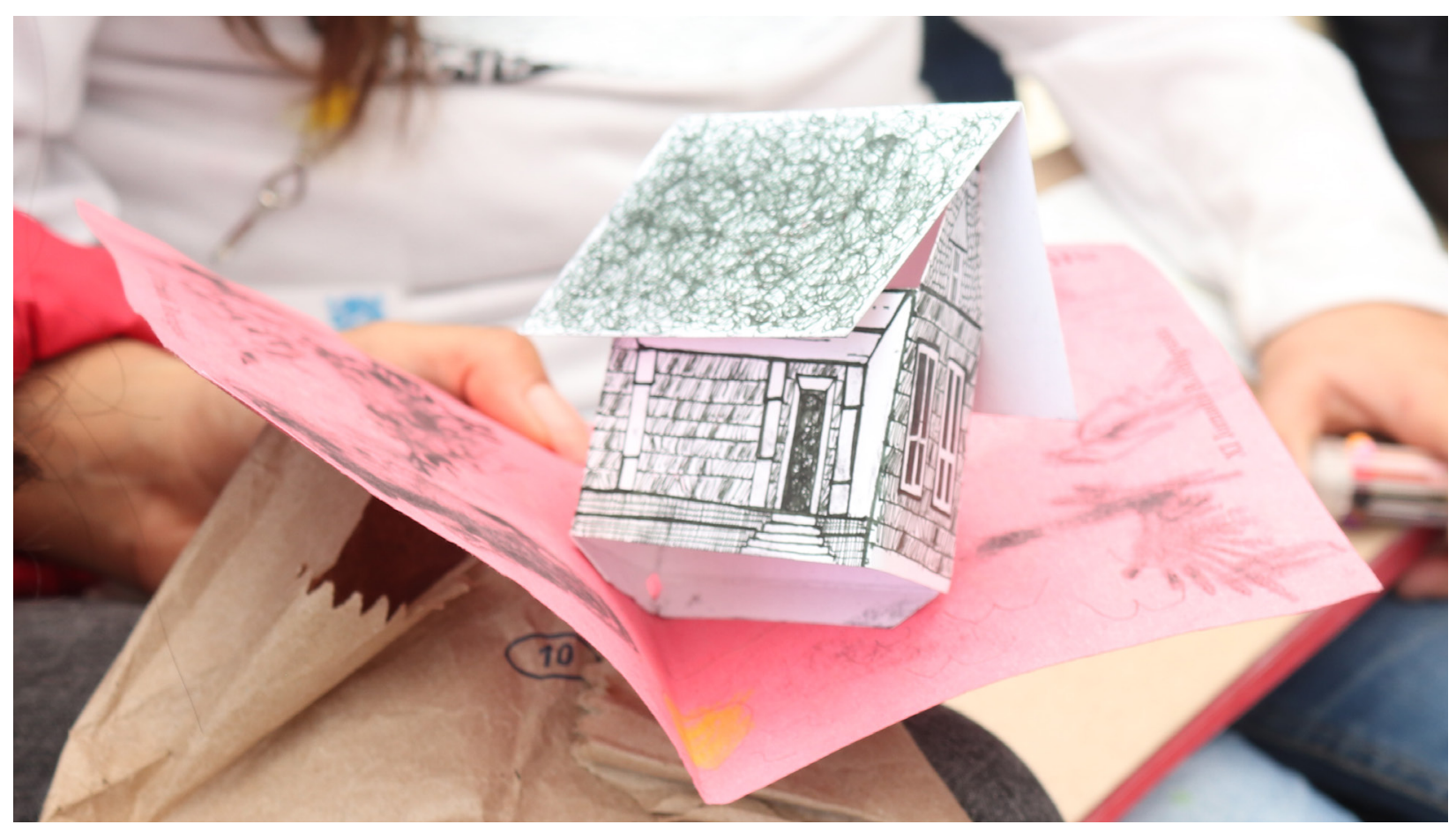

Fuente: Mitote ICDAC

Con esta encomienda realizamos la activación y le dimos seguimiento durante tres semanas. En este espacio aprendimos de Ixs estudiantes de cada aula, sin jerarquía de grados académicos, posicionándonos todxs nosotrxs no como estudiantes y docentes, sino como seres que aprenden juntxs, siendo rebeldes y liberadores, acorde a nuestra responsabilidad educativa, no solo de las normas institucionales, sino de la educación popular, la educación de este pueblo contemporáneo en el que vivimos y que nos implica. Ubicados desde donde hemos sido obligadxs a cumplir sistemáticamente estructuras de violencia educativa, en las que solo se ponderan la repetición y la cuantificación de conocimientos no significativos frente a un sistema que valida, o no, nuestro pensamiento en función de lo que podemos brindar a cierta parte de la sociedad, y que imposibilita el enunciar nuestras voces y construir posturas propias y colectivas.

Luego de las experiencias Rodriguistas, encontramos que ser rebeldes en un momento como este requiere postura y compromiso para sostenerse. Por ello, queremos plantear algunas consideraciones de postura al elaborar este texto. Comenzamos con un 
Pensar a Simón Rodríguez desde las prácticas artísticas:

Dossier

La indisciplina como una forma de resistencia afectiva

pensamiento muy transparente, definido, sólido y no por ello más sencillo de asumir con honesta conciencia crítica: escribimos desde la indisciplina como forma de resistencia afectiva, desde un lugar privilegiado, más privilegiado que muchas de las voces amigxs que han puesto, y continúan poniendo, Ix cuerpx para decir lo que se tiene que decir, desde la educación popular, desde las prácticas artísticas subversivas, desde el compartir saberes. También escribimos desde el sostener saberes en las comunidades y contextos que han tenido que resistir a partir de la auto-enunciación y la autogestión, aquellxs que desde la digna rabia han buscado ser reconocidxs y respetadxs dentro de un sistema que nos violenta todo el tiempo y desde todos los frentes. La digna rabia se encuentra en muchas formas de entender el enojo, la rabia que contiene una respuesta a un acto de injusticia repetido. Audre Lorde escribió en su texto Los usos de la rabia que:

My response to racism is anger. I have lived with that anger, on that anger, beneath that anger, on top of that anger, ignoring that anger, feeding upon that anger, learning to use that anger before it laid my visions to waste, for most of my life. Once I did it in silence, afraid of the weight of that anger. My fear of that anger taught me nothing. Your fear of that anger will teach you nothing, also. [Mi respuesta al racismo es la rabia. He vivido con esa rabia, en esa rabia, debajo de esa rabia, encima de esa rabia, ignorando esa rabia, alimentándome de esa rabia, aprendiendo a usar esa rabia antes de que arruinara mis visiones, durante la mayor parte de mi vida. Una vez lo hice en silencio, temiendo el peso de esa rabia. Mi miedo a esa rabia no me enseñó nada. Tu miedo a esa rabia no te enseñará nada]. (Lorde, 1997, p. 278)

De ahí que decimos esto como un ejercicio de consciencia que nos resulta necesario al ejercer la coherencia de nuestro hacer/pensar, con respecto de una perspectiva crítica. Decir esto no nos genera ninguna palmadita en la espalda, ni nos exime de ninguna responsabilidad, por el contrario, es un acto enunciativo desde el cual tomamos una postura y adquirimos un compromiso epistémico. Solemos pensar y cuestionarnos la situación en la que vivimos nuestra práctica educativa y lo ponemos aquí desde las palabras de bell hooks:

As an intellectual working as an academic I often felt that my commitment to radical openness and devotion to critical thinking, to seeking after truth, was at odds with the demands that I uphold the status quo if I wanted to be rewarded. My integrity was as much at risk in the academic world as it had been in the non-academic work world, where workers are expected to obey authority and follow set rules.

ESCENA. Revista de las artes, 2021, Vol. 81, Núm. 1 (julio-diciembre), pp. 282-303 
While much lip service is given to the notion of free speech in academic set- tings, in actuality constant censorship-often self-imposed - takes place. Teachers fear they will not receive promotions or that in worst-case scenarios they will lose their jobs. Even so, in our society the academic world remains the primary place where teaching and learning are valued, where reading and thinking are deemed meaningful and necessary work. This validation, however limited in scope, provides affirmation and sustenance for academics and/or intellectuals in an anti-intellectual culture. [Como intelectual que trabajaba como académica, a menudo sentía que mi compromiso con la apertura radical y la devoción al pensamiento crítico, con la búsqueda de la verdad, estaba en desacuerdo con las demandas de mantener el status quo si quería ser reconocida. Mi integridad estaba en riesgo tanto en el mundo académico como en el mundo laboral no académico, donde se espera que los trabajadores obedezcan a la autoridad y sigan las reglas establecidas. Si bien se habla mucho de la noción de libertad de expresión en el ámbito académico, en realidad se produce una censura constante, a menudo autoimpuesta. Los profesores temen no recibir ascensos o que, en el peor de los casos, perderán sus trabajos. Aun así, en nuestra sociedad el mundo académico sigue siendo el lugar principal donde se valora la enseñanza y el aprendizaje, donde la lectura y el pensamiento se consideran un trabajo significativo y necesario. Esta validación, aunque de alcance limitado, proporciona afirmación y sustento a los académicos y/o intelectuales en una cultura antintelectual]. (2003, p. 22)

A partir de esta declaración de bell hooks, debemos decir que escribimos desde la Universidad Nacional Autónoma de México, desde la Facultad de Artes y Diseño, desde la Academia, desde la Universidad Pública, desde esas estructuras que, con tristeza y rabia, debemos asumir que han sido configuradas como un instrumento de regulación ideológica, como una herramienta de control sobre las subjetividades, como un espacio que nos alecciona para ser sujetos desde la violencia sistémica y estructural. Si bien ha sido todo esto, es importante decir que la Universidad también ha sido un espacio para el encuentro, como dice bell hooks, un espacio de sustento, un espacio posibilitador de diálogos y resistencias, un ambiente articulador de comunidades que hemos aprendido a mirar las brechas del sistema como un territorio para detonar en colectivo. Es un lugar-nodo desde el cual nos hemos permitido deshacernos del yo, en pos de un nosotros electivo, el lugar desde el que hemos articulado la resistencia del Mitote ICDAC. 
Pensar a Simón Rodríguez desde las prácticas artísticas:

Imagen 7. Mitoteando el punto/ Mitote Simón Rodríguez

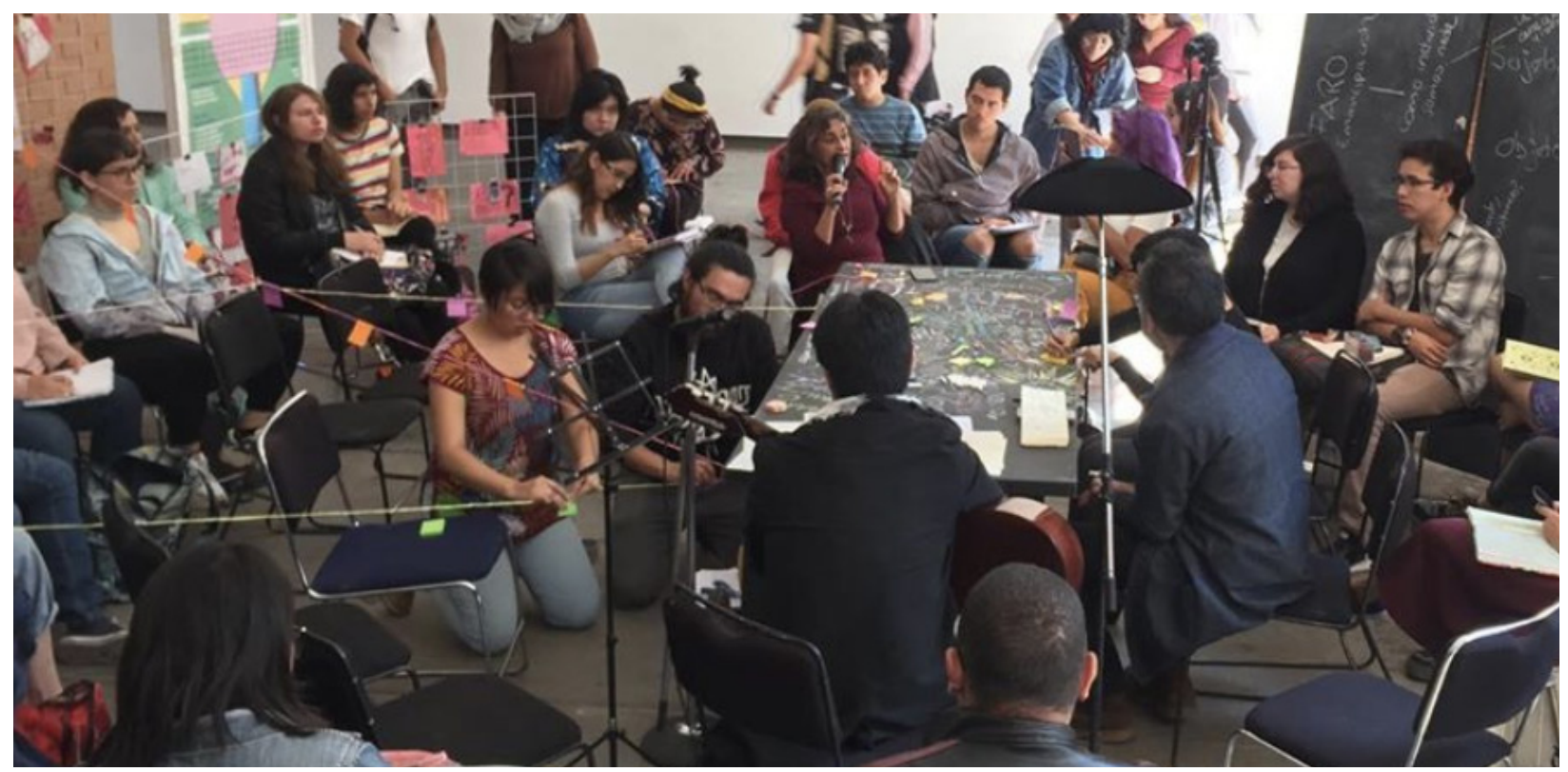

Fuente: Mitote ICDAC

Sin embargo, no debemos olvidar que es nuestra responsabilidad el hacer factible la existencia de espacios donde sea posible y necesario reflexionar, de manera seria, sobre nuestras acciones pedagógicas situadas. Los espacios que existen no han sido pensados como lugares desde los cuales habitar la idea de compartir saberes ni desde la hospitalidad del pensamiento. No hemos logrado aún articular espacios reflexivos transversales y tejidos. Con esto, hemos dejado de lado la vocación popular que nos permitiría ver, en la práctica educativa, una práctica de libertad, y de manera particular, desde las prácticas artísticas, una herramienta para el ejercicio de la libertad estética, una estética de la liberación, diría Dussel (2020).

\section{Resistir, insistir, persistir.... es más fácil cuando se acuerpa desde voces amigas}

Simón Rodríguez, el filósofo tipógrafo, el filósofo andante, el zambo loco, es una de esas voces entrañables desde las cuáles nos hemos acuerpado, no solo como un personaje histórico localizado en la América política, territorial y militarmente colonizada por Europa, sino como un ser situado en un mundo brutalmente configurado desde las lógicas coloniales en pleno siglo XIX. Sufrió múltiples tipos de violencia, tanto física como intelectualmente, 
a lo largo de su vida, dinámica que tristemente nos hermana con él y sus ideas a casi dos siglos de distancia, pues las experiencias de violencia estructural que han modelado nuestra América, siguen siendo vigentes en el actual siglo. También nos vinculamos desde su condición de expósito, de niño abandonado, y el hecho de que, desde su propia vida e imagen, se vio como un criptógamo. Rodríguez vivió el racismo, la discriminación y los embates del borramiento histórico y llamaba criptógamos a:

seres que habían venido al mundo de una semilla que no llevaba los nutrientes necesarios para crecer. Seres necesitados de ayuda para terminar de nacer. De allí una metáfora fundamental de Sociedades americanas en 1828, su obra principal: la fundación de repúblicas auténticas, la construcción de un "pueblo», no es solo una ficción formal: acontece cuando la gente más desprotegida ha adquirido capacidades intelectuales para disputar la verdad y el saber, y condiciones materiales para cultivar colectivamente su vida sin tener que pedirle permiso a los más fuertes. (Mondragón Velázquez, 2020, párr. 3)

"Se resiste a lo que hay desde lo que hay" (Canal UCI Red, 2014, 2m 31s), dice Benjamin Berlanga en su ponencia Educar con sujeto: La Educación como Resistencia. Por ello, enunciamos, desde lo que hay, nuestra resistencia, así en plural, en comunitario electivo, como aquello que dice, de manera frontal, no a la normalización y ocultamiento de la idea y práctica de un sistema educativo que no solo se aleja de la idea de la vida buena y el bien vivir, sino que genera, perpetua y defiende lógicas que instauran una pedagogía de la crueldad, desde un amplio catálogo de violencias siempre disimuladas; pedagogía que se pretende, además, sea abrazada sin miramientos ni cuestionamientos. Las palabras de Berlanga nos resuenan mucho al leer a Rodríguez, con la sugerencia de acciones para comprender y comprendernos para poder entender bajo qué parámetros generalizados se nos está moldeando y resistirlos, ¿cómo hacerlo? Pensamos que eso se construye en colectivo y encaminado al constante cuestionamiento de lo establecido, sabiéndonos como sujetos afectados y afectivos. Tal como menciona María del Rayo Ramírez Fierro, "no hay acciones sin sujetos de la acción o agentes, y como se trata de sujetos vivos, son sujetos con emociones que, incluso gestualizan y/o corporizan" (citado en Rawicz, 2020, p. 82). Con las palabras de nuestra querida colega encontramos sur para resistir afectivamente desde las acciones a las que somos sujetxs, aquellas que nos implican y nos hacen acuerpar para comprender, proponer, compartir, diferir y aprender desde lo que vemos en aquellas acciones, sus circunstancias y lo que las rodea. 
Pensar a Simón Rodríguez desde las prácticas artísticas:

Dossier

La indisciplina como una forma de resistencia afectiva

Quienes cuestionan y disienten desde estos espacios, que buscan sobre todo la obediencia y la homogenización del pensamiento, es llamado y percibido como incómodo. Somos aún más incómodxs si nos narramos en la primera persona del plural y decimos esto como una declaración humilde y que nos sitúa nunca como una forma de justificarnos ni para dejar de poner el cuerpo, sino todo lo contrario. Creemos que todxs participamos en el sistema, pero hay formas de salir de él, en palabras de bell hooks:

Every black person and person of color colludes with the existing system in small ways every day, even those among us who see ourselves as anti-racist radicals. This collusion happens simply because we are all products of the culture we live within and have all been subjected to the forms of socialization and acculturation that are deemed normal in our society. Through the cultivation of awareness, through the decolonization of our minds, we have the tools to break with the dominator model of human social engagement and the will to imagine new and different ways that people might come together. [Todas las personas negras y de color se coluden con el sistema existente en pequeñas formas todos los días, incluso aquellxs entre nosotrxs que nos vemos como radicales antirracistas. Esta colusión ocurre simplemente porque somos productos de la cultura en la que vivimos y todxs hemos sido sometidxs a las formas de socialización y aculturación que se consideran normales en nuestra sociedad. A través del cultivo de la conciencia, a través de la descolonización de nuestras mentes, tenemos las herramientas para romper con el modelo dominante del compromiso social humano y la voluntad de imaginar nuevas y diferentes formas en que las personas pueden unirse]. (hooks, 2003, p. 36)

Las voces amigas que nos han acompañado a través de los siglos y hasta ahora, mediante las letras y palabras, nos han dado cobijo en tiempos convulsos, pandémicos y llenos de miedos. Abrazamos a esas voces, abrazamos a quienes nos han acompañado tejiendo redes, lazos y resistencias, a quienes han construido con nosotrxs refugio cuando el peso de las violencias hegemónicas ha intentado hacernos retroceder para obligarnos a soltar la responsabilidad de seguir poniendo el cuerpo, para decir lo que se tiene que decir, para seguir acuerpando lo dicho por nuestros amigos en situaciones que han buscado destruir la dignidad y la autonomía de los espíritus que, antes y ahora, han puesto su vida para resistir frente a los discursos dominantes. Estas voces, acuerpamientos y el acto de enunciarles son un acto reflexivo de indisciplina dentro de un largo legado de control y gestión de masas. Es un reflejo de nuestras prácticas al construir, desde otras trincheras, 
un espacio de resistencia afectiva que crea voluntades. Tal como pensaba y hacía el buen Simón o como también nos permitimos pensar con bell hooks quin, quien dice:

To build community requires vigilant awareness of the work we must continuaIly do to undermine all the socialization that leads us to behave in ways that perpetuate domination. A body of critical theory is now available that explains all the workings of white-supremacist thought and racism. But explanations alone do not bring us to the practice of beloved community. When we take the theory, the explanations, and apply them concretely to our daily lives, to our experiences, we further and deepen the practice of anti-racist transformation. [Construir comunidad requiere una conciencia vigilante del trabajo que debemos hacer continuamente para quebrar la socialización que nos lleva a comportarnos de maneras que perpetúan la dominación. Ahora se dispone de un cuerpo de teoría crítica que explica el funcionamiento del pensamiento y el racismo de la supremacía blanca. Pero las explicaciones por sí solas no nos llevan a la práctica de la comunidad amorosa. Cuando tomamos la teoría, las explicaciones y las aplicamos de manera concreta a nuestras experiencias, extendemos y profundizamos en la práctica de la transformación antirracista]. (hooks, 2003, p. 36).

\section{No creemos en conclusiones, pero si en cierres narrativos}

Hay que entender que nunca estamos solxs cuando ponemos ideas en soportes, porque nunca somos solo nosotrxs al hablar. Insertarnos en la consigna de que hay una propiedad privada de las ideas es una violencia que hemos normalizado y ocultado bajo múltiples discursos de eficiencia y productividad, pero que nos atraviesa a todxs. Esto atomiza la resistencia y la hospitalidad del saber y, a la vez, nos impide construir refugios epistémicos desde las múltiples miradas que constituyen el mundo nuestro, lo cual pondera únicamente la exclusividad de clases sociales. Pero nosotrxs tampoco creemos en eso, pues si realmente ocurriera no estaríamos hablando desde una universidad pública, ni mucho menos desde una América rebelde que sabe de quienes la construyen y lo que tenemos que decir a partir de una educación popular que sí ve por una sociedad y las acciones que de ella derivan naturalmente. En palabras de Rodríguez: "el objeto de la INSTRUCCION es la SOCIABILIDAD / i el...... de la Sociabilidad es hacer menos penosa la vida". (Rodríguez, 1851/2018, p. 61)

Esta última línea nos hace reflexionar profundamente sobre las prácticas artísticas. Notamos que hacen falta refugios desde los cuales podamos sostener y dejarnos sostener; 
Pensar a Simón Rodríguez desde las prácticas artísticas:

Dossier

La indisciplina como una forma de resistencia afectiva

escuchar y escucharnos, y ejercitar la elección determinando un pensamiento crítico respecto a nuestros afectos. El hacer comunidad afectiva es siempre un acto electivo, en todas las direcciones, el cual requiere confianzas y justicias epistémicas que nos involucren como sujetos constructorxs de saberes comunitarios, no solo desde lo electivo, sino desde nuestro genuino derecho que posibilita también el bien vivir. Este último entendido como un acto secretamente negado a las prácticas académicas, tal vez por miedo a la vulnerabilidad que implica sabernos en una posición de inevitable relación con el otro, ese otro al que nos han presentado como el ajeno, el enemigo, quien implica el riesgo de hacernos mal. Este miedo incrustado en los discursos hegemónicos (jamás aceptado, pero siempre enunciado y visible a ojos críticos), puede que esté sustentado en una sencilla premisa: sería caótico para un sistema basado en la otrificación, entendida por Antonio Corona como:

un acto de violencia simbólica, un ejercicio de poder sobre el otro para situarlo desfavorablemente en comparación con la propia identidad. De manera más precisa, llamamos otrificación a la negación de la complejidad humana en aquellas personas que categorizamos como ajenos a nuestros grupos identitarios, y su reducción a características definitorias que, generalmente, calificamos como indeseables. (2018, p. 49)

Apuntamos al descubrimien- Imagen 8. Cucharadas de verdad / Leer a Simón to de que juntxs y desde el nosotrxs podamos, como dice Simón Rodríguez en la portada de la edición de Arequipa de Sociedades Americanas en 1828, tenemos la potestad de definir e imaginar "cómo serán y como podrían ser los siglos venideros" (Rodríguez, 2018, parr.1), y mirar con claridad que "en esto han de pensar los americanos, no en pelear unos con otros". (Rodríguez, 2018, parr.3)

La intención de Rodríguez, la nuestra y la de muchxs colegas más está basada en la idea de la educación popular para la reflexión y conciencia social. También basada en una

\section{Rodríguez}

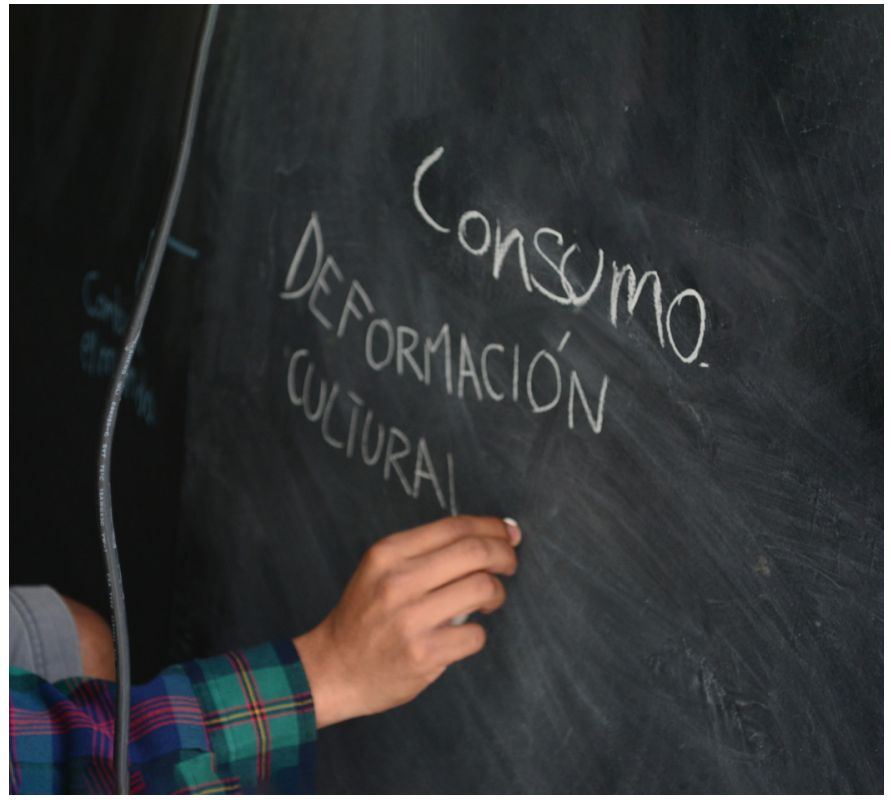

Fuente: Mitote ICDAC 
educación popular que abogue por la decolonización de la vida, por las causas sociales, y que haga frente al dominio insensible y violento. Este dominio que ha sido basado en la oposición de Ixs privilegiados enceguecidos por la jerarquía de las clases sociales y económicas; en los gobiernos escandalizados por la duda de las formas establecidas; en la impuesta cultura colonial, la cual hemos arrastrado por siglos, y en el rotundo rechazo de las propuestas y prácticas educativas que sí responden a las necesidades del bien vivir.

A partir de todo lo anterior, pensamos y trabajamos desde aquella indisciplina que nota, como dice Grecia Monroy, una "contraposición [que] corresponde a la tensión entre una acción político-dominadora y una acción político-revolucionaria” (citada en Rawicz, 2020, p. 115). A partir de estas acciones distinguimos a la acción primera y en voz de Freire aquella que "no supone esta dimensión con la misma necesidad pues la propia funcionalidad mecánica e inconsciente de la estructura es mantenedora de sí misma y por lo tanto de la dominación" (Fiori, citado en Freire, 2005, p. 50). Podemos ver que estas palabras aún son vigentes y, por ello, es indiscutiblemente necesario replantear todo; todo aquello que representa hegemonía, violencia de cualquier tipo y las estructuras binarias que incitan a la validación falsa del deber ser. Por el contrario, vemos y somos partidarixs de la acción político-revolucionaria como acto de libertad, consciente, sensible, electivo y afectivo que nombra al sujeto en y a partir de sus contextos, aquel que vive y propicia el diálogo para la educación. Una acción en la que juntxs construyamos la realidad y con ella la historia.Fue dicho el 13 de diciembre del año 2020 (año de la pandemia por Covid-19 en la Ciudad de México) 5 .

5 Como una forma de desobediencia epistémica y de rechazo a las formas que norman y perpetúan las nociones de propiedad privada sobre las enunciaciones y los discursos comunitarios, esta nota quiere dejar constancia de que en el MITOTE-ICDAC, siempre que nos es posible, enunciamos nuestra renuncia a la idea de autoría como forma de propiedad sobre las ideas y las palabras que las acuerpan, pues creemos que toda construcción discursiva en buena medida comunitaria y resultado de muchas interacciones reflexivas y afectivas, por ello en esta nota enunciamos que Rubén Cerrillo Mitote, Adriana Raggi Mitote y María Sanes Mitote, hemos confabulado.

ESCENA. Revista de las artes, 2021, Vol. 81, Núm. 1 (julio-diciembre), pp. 282-303 
Pensar a Simón Rodríguez desde las prácticas artísticas:

Dossier

La indisciplina como una forma de resistencia afectiva

\section{Referencias}

Afrontera. (28 de octubre de 2020) Ternura radical en tiempos de colonialidad y transfobia. [Facebook Watch]. Recuperado de: https://www.facebook.com/105205084625580/videos/371496117402116/

Canal UCI Red. (2 de mayo de 2014). Educar con sujeto: La Educación como Resistencia. 2 do Coloquio Construcción Utópica y lucha social. [Video de Youtube]. Recuperado de https://www.youtube.com/watch?app=desktop\&feature=youtu.be\&v=O3pUg4FOoHM

Dussel, E. (13 de mayo de 2020). Curso: Estética de la Liberación latinoamericana. Clase 2. [Video de Youtube] Recuperado de https://www.youtube.com/watch?v=mYW1d8lz18U

Corona, A. (2018). Mecanismos de otrificación entre la oposición política en Twitter durante las elecciones estatales de 2017 en México. adComunica. Revista Científica de Estrategias, Tendencias e Innovación en Comunicación, 1(16), 45-70. http://dx.doi. org/10.6035/2174-0992.2018.16.4

D’Emilia, D., \& Chávez, D. B. (16 de julio 2015). Ternura radical es.... hysteria revista, 14(fluidos). Recuperado de https://hysteria.mx/ternura-radical-es-manifiesto-vivo-por-dani-demilia-y-daniel-b-chavez/

Freire, P. (2005). Pedagogía del oprimido. México: Siglo XXI. (Publicación original 1975).

hooks, b. (2003). Teaching community. A Pedagogy of Hope. Nueva York, Estados Unidos: Routledge.

Illich, I. (2006). Obras Reunidas I. México: Fondo de Cultura Económica.

Lorde, A. (1997). The Uses of Anger. Women's Studies Quarterly. 25, (1 y 2), 278-285.

Mondragón Velázquez, R. (28 de octubre 2020). Simón Rodríguez, demonio de los mesones. Jacobin. América Latina, 1. Recuperado de https://jacobinlat.com/2020/10/28/ simon-rodriguez-demonio-de-los-mesones/

Rawicz, D.(Coord.). (2020). Leer a Simón Rodríguez. Proyecto para la América. México: Universidad Autónoma de la Ciudad de México.

ESCENA. Revista de las artes, 2021, Vol. 81, Núm. 1 (julio-diciembre), pp. 282-303 
Rodríguez, S. (2018) Sociedades Americanas en 1828. México: Universidad Autónoma Metropolitana. (Publicación original de 1828)

Zubiri, X. (1993). Sobre el sentimiento y la volición (2a ed.). México: Alianza. 